\title{
International Neuroblastoma Staging System
}

National Cancer Institute

\section{Source}

National Cancer Institute. International Neuroblastoma Staging System. NCI Thesaurus.

Code C85416.

A surgicopathological staging system for neuroblastoma, based on the Evans staging system. 